\title{
Fracture Roughness Scaling: a case study on planar cracks
}

\author{
Stéphane Santucci, ${ }^{1,2,3}$ Mélanie Grob, ${ }^{4}$ Alex Hansen, ${ }^{5}$ Renaud \\ Toussaint, ${ }^{4}$ Jean Schmittbuhl, ${ }^{4}$ and Knut Jørgen Måløy ${ }^{3}$ \\ ${ }^{1}$ Laboratoire de physique, UMR CNRS 5672, Ecole Normale Supérieure de Lyon, 46 allée d'Italie, 69007 Lyon, France \\ ${ }^{2}$ Physics of Geological Processes, Oslo University, 1048 Blindern, N-0316 Oslo, Norway \\ ${ }^{3}$ Fysisk Institutt, Universitetet $i$ Oslo, 1048 Blindern, N-0316 Oslo, Norway \\ ${ }^{4}$ Institut de Physique du Globe de Strasbourg, UMR CNRS 7516, \\ EOST / Université de Strasbourg, 5 rue René Descartes, F-67084 Strasbourg Cedex, France \\ ${ }^{5}$ Institutt for fysikk, Norges teknisk-naturvitenskapelige Universitet, N-7491 Trondheim, Norway
}

(Dated: May 11, 2018)

\begin{abstract}
Using a multi-resolution technique, we analyze large in-plane fracture fronts moving slowly between two sintered Plexiglas plates. We find that the roughness of the front exhibits two distinct regimes separated by a crossover length scale $\delta^{*}$. Below $\delta^{*}$, we observe a multi-affine regime and the measured roughness exponent $\zeta_{\|}^{-}=0.60 \pm 0.05$ is in agreement with the coalescence model. Above $\delta^{*}$, the fronts are mono-affine, characterized by a roughness exponent $\zeta_{\|}^{+}=0.35 \pm 0.05$, consistent with the fluctuating line model. We relate the crossover length scale to fluctuations in fracture toughness and the stress intensity factor.
\end{abstract}

PACS numbers: $62.20 . \mathrm{Mk}, 46.50 .+\mathrm{a}, 68.35 . \mathrm{Ct}$

Since the pioneering work of Mandelbrot et al. [1] demonstrating the self-affine character of fracture surfaces of metals, numerous studies have been devoted to the morphology of fracture surfaces [2, 3]. In particular, the roughness exponent $\zeta_{\perp}$ characterizing this selfaffinity was shown to be very robust and further on conjectured to be universal [4] with $\zeta_{\perp} \sim 0.8$ over a large set of materials and conditions [2 -5] and up to very large scales [6]. In the weak disorder limit when toughness fluctuations are small compared to stress loading fluctuations, there are data suggesting that $\zeta_{\perp}$ takes on a smaller value, 0.4 [7, 8]. A first attempt at investigating the origin of a universal fracture roughness exponent in the quasistatic propagation limit was made by Hansen et al. [9] who suggested that in two dimensions it might be related to the directed polymer problem. This idea was further developed by Räisänen et al. [10]: the fracture surface follows the surface that minimizes the integrated strength of the intact material. Numerical studies based on this idea gave a roughness exponent $\zeta_{\perp}=0.41 \pm 0.02$. A different idea was proposed by Bouchaud et al. [11]. In their picture, the fracture surface is the "footprint" of a passing fluctuating elastic line - the fracture front - moving through a disordered three-dimensional landscape. This powerful idea opened up for the existence of two roughness exponents: one describing the roughness orthogonal to the average crack plane $\zeta_{\perp}$ and another describing the roughness of the front in the average roughness plane $\zeta_{\|}[12]$.

In order to simplify the 3-d configuration, Schmittbuhl et al. [13] proposed a numerical model where the crack front is constrained to propagate along a weak plane (suppressing the out-of-plane roughness). The in-plane roughness exponent was found equal to $\zeta_{\|}=0.35$, and later on, refined to $\zeta_{\|}=0.39$ by Rosso and Krauth [14]. Even

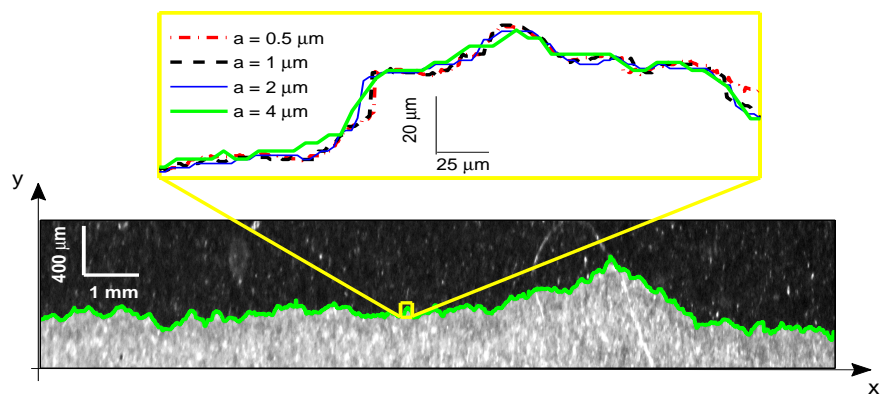

FIG. 1: A typical fracture (using glass beads of a diameter $\varnothing_{1} \sim 50 \mu \mathrm{m}$ for roughening the samples) and a zoom of the crack front $y(x)$ to emphasize the effect of the optical resolution given by the pixel size $a$.

though such a fluctuating line approach [15] can match several scaling exponents related to the crack front dynamics [16], it fails at reproducing the in-plane roughness exponent $\zeta_{\|}$measured up to now around $0.6[17-19]$. We will adress precisely this problem in the present Letter, insisting on the fact that the fluctuating line model is typically a perturbative approach assuming the local slope of the front to be small, and ignoring crack coalescence [20]. A theory based on a mapping of the fracture process to a correlated percolation one [21, 22] considered precisely the latter aspect. Numerical simulations based on this model gave $\zeta_{\|}=0.6$, substantially larger than the value found based on the fluctuating line model but consistent with the experimental results obtained up to now. The coalescence model also clarified the controversy over the concept of self-affinity 23 25].

The goal of the present Letter is to address the possible coexistence of two roughness scaling regimes in the case of in-plane fracture. We analyze stable mode I fracture 
fronts propagating along the sand blasted and sintered contact plane between two PMMA plates [16 19, 26]. An important contribution to our analysis comes from the compilation of numerous observations at different resolutions. We observe that the planar cracks follow two distinct scaling regimes, multi-affine at small scales and mono-affine at larger ones, characterized by different roughness exponent $\zeta_{\|}^{-}=0.60$ and $\zeta_{\|}^{+}=0.35$, respectively. We show that those two regimes are separated by a well-defined crossover length $\delta^{*}$ controlled by a balance between the stress intensity factor variability along the fracture front and fluctuations in the fracture toughness. Below $\delta^{*}$, the value of the roughness exponent is in agreement with the prediction of the coalescence model $\zeta_{\|}=0.60$, while the large-scale exponent is consistent with the value predicted by the fluctuating line model, $\zeta_{\|}=0.39$. We argue in the second part of the manuscript why both theories may be correct in describing the experiment, but operating at different length scales.

Experiments -The experimental setup allows for a stable mode I crack propagation along a weak plane in a PMMA block from a displacement imposed normally [17]. Toughness fluctuations along the weak plane are artificially introduced during sample preparation, which consists of annealing two sandblasted PMMA plates. In order to modify the toughness fluctuations, we changed the type and the size of the blasting particles using glass beads of diameters around $\varnothing_{1} \sim 50 \mu \mathrm{m}, \varnothing_{2} \sim 200 \mu \mathrm{m}$, and $\varnothing_{3} \sim 300 \mu \mathrm{m}$ and a glass-aluminum powder with a typical particle size around $S \sim 50 \mu \mathrm{m}$. We also changed the loading speed and procedure, with interfaces recorded during their propagation at various velocities. In order to obtain a multi-high resolution description of the fronts, we considered fracture fronts at rest. During those experiments, after a slow crack propagation, the sample was unloaded in order to arrest the crack. Then, we took high resolution pictures $(3871 \times 2592$ pixels $)$ of the front at rest (Fig. 1) using a digital camera mounted on an optical microscope. Using a translation stage that can move the microscope in the $x$ direction parallel to the front (and perpendicular to the fracture propagation direction $y$ ) neighboring pictures were taken. Up to 15 high resolution pictures were then assembled resulting in fracture fronts with around 25000 data points and a pixel size $a=0.48 \mu \mathrm{m}$. To remove acquisition artifacts, different resolutions of the front description were obtained by changing the magnification of the optical zooms (see Fig. (1). This results in images of the same fracture at resolutions, $4,2,1$ and $0.48 \mu \mathrm{m}$ per pixel with respectively around $4000,8000,16000$ and 25000 data points per image. This procedure was repeated 20 times in order to obtain 20 independent fracture fronts. The actual total length of each analyzed crack was around $15 \mathrm{~mm}$.

Two scaling regimes - We analyze the height fluctuations of the crack fronts $\Delta y(\delta)=y(x+\delta)-y(x)$ where $y(x)$ is the advance of the front along the $y$ direction at

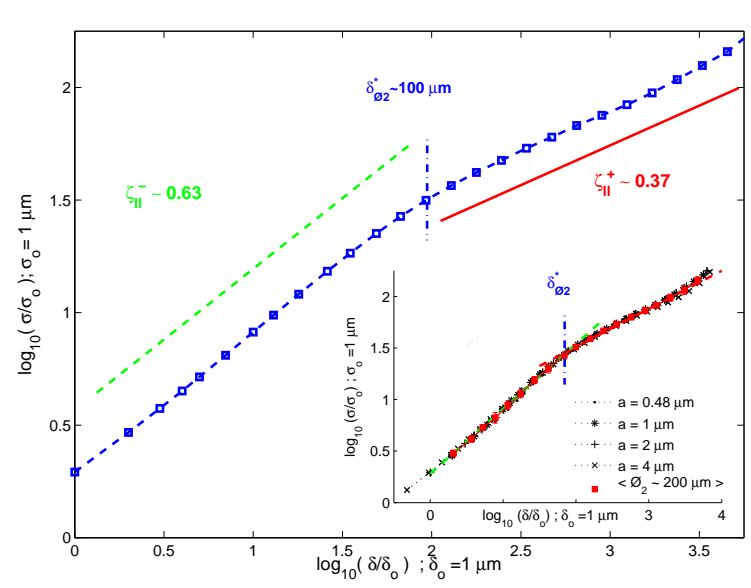

FIG. 2: Scaling behavior of the rms $\sigma$ of the height fluctuations $\Delta y(\delta)$ with two different roughness exponents $\zeta_{\|}^{-} \sim 0.63$ and $\zeta_{\|}^{+} \sim 0.37$, below and above the typical scale $\delta^{*} \sim 100 \mu \mathrm{m}$ respectively. The inset shows that this scaling is independent of the image resolution $a$.

position $x$. We will first consider fracture fronts at rest for samples prepared with $200 \mu \mathrm{m}$ glass beads. Then, we will prove the universality of our results showing the same analysis for various experimental conditions. In Fig. 2 , we examine the scaling behavior of the root mean square (rms) of the height fluctuations $\sigma(\delta)=\left\langle\Delta y^{2}(\delta)\right\rangle^{1 / 2}$. At small scales below $\delta^{*} \sim 100 \mu \mathrm{m}$, we observe a self-affine scaling behavior: $\sigma(\delta) \propto \delta^{\zeta_{\|}^{-}}$with a roughness exponent $\zeta_{\|}^{-}=0.60 \pm 0.05$. This is consistent with previous experimental measurements [16 19] and the value predicted by the coalescence model [22]. However, at scales larger than $\delta^{*}$, we observe a crossover to another scaling regime with a smaller roughness exponent $\zeta_{\|}^{+}=0.35 \pm 0.05$. This value corresponds to the fluctuating line model prediction $\zeta_{\|}=0.39$ [14]. Due to the limited scaling range for $\delta>\delta^{*}$, our data do not rule out a possible slow crossover to a flat front (no disorder regime), at large scales 32]. In the inset of Fig. 2. we demonstrate the robustness of our results by showing that the two different scaling regimes and the cross-over length scale observed are independent of the sampling resolution of the interface.

In order to study in more details those different scaling behaviors, we develop a multi-scaling analysis [26] by performing a direct measurement of the pdf $P\left[\Delta^{\prime} y\right]$ of the height fluctuations $\Delta^{\prime} y(\delta)=\Delta y(\delta)-\langle\Delta y\rangle$ and computing their structure functions $C_{k}(\delta)=\left\langle|\Delta y(\delta)|^{k}\right\rangle_{x}^{1 / k}$. On Fig. 3, we show the distributions of the height fluctuations $P\left[\Delta^{\prime} y(\delta)\right]$ for logarithmically increasing length scales $\delta$. Above the characteristic length scale $\delta^{*} \sim 100$ $\mu \mathrm{m}$, the shape of the distributions is Gaussian, while for smaller length scales, we observe long tails consistent with a non-Gaussian and multi-affine scaling. Indeed, we show that the structures functions $C_{k}(\delta)$-when normal- 


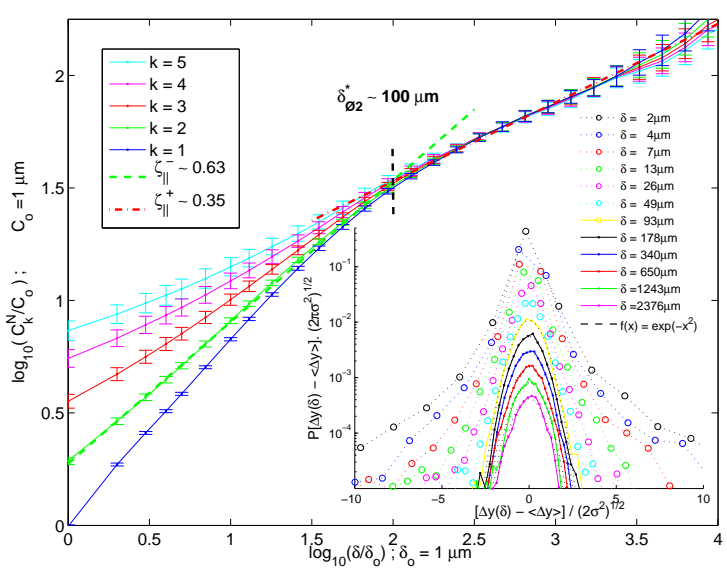

FIG. 3: We observe two different scaling regime of the normalized structure functions $C_{k}^{N}(\delta)=C_{k}(\delta) / R_{k}^{G}(\delta)$ separated by the cross-over $\delta^{*} \sim 100 \mu \mathrm{m}$. The corresponding p.d.f. of the height fluctuations $\Delta y(\delta)$ are shown in inset where we plot in semi- $\log P\left[\Delta^{\prime} y(\delta)\right] \cdot \sqrt{2 \pi \sigma^{2}}$ vs. $\left[\Delta^{\prime} y(\delta)\right] / \sqrt{2 \sigma^{2}}$ for increasing length scales $\delta$, shifted vertically for visual clarity. Above $\delta^{*}$, the lines $f(x)=e^{-x^{2}}$ fit the parabolic shape of the Gaussians.

ized by the set of values $R_{k}^{G}=\sqrt{2}\left(\Gamma\left(\frac{k+1}{2}\right) / \sqrt{\pi}\right)^{1 / k}$ corresponding to the ratios $R_{k}^{G}=C_{k}^{G}(\delta) / C_{2}^{G}(\delta)$ obtained for a Gaussian and mono-affine signal (see [26] for details)collapse and follow a self-affine scaling with a unique roughness exponent $\zeta_{\|}^{+}=0.35 \pm 0.05$ corresponding to the elastic line prediction. Below $\delta^{*}$, the fanning of the structure functions confirms the deviation to the Gaussian statistics and reveals an effective multi-affine behavior consequence of the heterogeneities along the interface leading to steep crack front slopes. A fit to $C_{2}(\delta)$ on that range gives $\zeta_{\|}^{-}=0.60 \pm 0.05$, in agreement with the coalescence model [22].

Cross-over length scale - We now investigate what controls the crossover length $\delta^{*}$. We base our discussion on the Griffith criterion that assumes a balance between the stress intensity factor $K$ and the fracture toughness, $K_{c}$. We introduce a mean field argument to describe the stress intensity factor variation in the direction of the propagation around the average position of the front $\bar{y}: K(y)=$ $K_{0}(\bar{y})+K^{\prime}(y-\bar{y})$ where $K^{\prime}=\partial K / \partial y$ is the average local gradient of the stress intensity factor. Then, the toughness of the asperities along the weak plane is supposed to be random around an average $K_{c}^{*}$ and uncorrelated beyond the asperity size $\delta_{c}$. We assume that $K_{0}(\bar{y})=K_{c}^{*}$ and that the fluctuation of the toughness over the front width $\sigma\left(\delta_{c}\right)$ reads as: $K_{c}\left(\bar{y} \pm \sigma\left(\delta_{c}\right)\right)=K_{c}^{*} \pm \Delta K_{c}\left(\delta_{c}\right)$ where $\Delta K_{c}$ is the magnitude of the toughness fluctuations on scale equal or larger than $\delta_{c}$. Finally, we estimate the width of the crack front $\sigma\left(\delta_{c}\right)$ to be the typical scale in the $y$-direction at which the failure criterion is met: $K\left(\bar{y}+\sigma\left(\delta_{c}\right)\right) \approx K_{c}\left(\bar{y}+\sigma\left(\delta_{c}\right)\right)$. Hence at a first order, we get an estimate of the front width at the asperity scale as a function of the magnitude of the toughness fluctuations $\Delta K_{c}$ and the local stress gradient $K^{\prime}: \sigma\left(\delta_{c}\right)=\Delta K_{c} / K^{\prime}$. Due to the self-affinity of the front with a roughness exponent $\zeta_{\|}$, our argument leads to an estimate of the prefactor of this scaling as:

$$
\sigma(\delta)=\sigma\left(\delta_{c}\right)\left(\frac{\delta}{\delta_{c}}\right)^{\zeta_{\|}}=\left(\frac{\Delta K_{c}}{K^{\prime}}\right)\left(\frac{\delta}{\delta_{c}}\right)^{\zeta_{\|}} .
$$

An important consequence is that the scaling of the fracture front will be hidden in the no disorder limit: when either the toughness disorder disappears $\left(\Delta K_{c} \rightarrow 0\right)$ or when the loading gradient becomes very large $\left(K^{\prime} \rightarrow \infty\right)$.

We now address the estimate of the local slope of the crack front at the asperity scale, i.e., $\sigma\left(\delta_{c}\right) / \delta_{c}$. Two cases emerge. First, if the local slope is small, $\sigma\left(\delta_{c}\right) \ll \delta_{c}$ the front may be described using a perturbative approach. We expect in this case that the fluctuating line model to be valid, leading to a roughness exponent $\zeta_{\|}^{+} \approx 0.39$. We note here that $\sigma(\delta) \ll \delta$ is valid for all $\delta>\delta_{c}$ if it is fulfilled for $\delta_{c}$ due to the self affinity of the front. The second situation occurs when $\sigma\left(\delta_{c}\right) \geq \delta_{c}$. In this case, we assume the coalescence model to be valid with a roughness exponent $\zeta_{\|}^{-}=0.6$. Hence, the slope at a scale $\delta$ scales as $\sigma(\delta) / \delta \propto \delta^{\zeta_{\|}^{-}-1}$, which means that it decreases with increasing $\delta$. This implies that there is a scale $\delta^{*}$ at which the slope $\sigma\left(\delta^{*}\right) / \delta^{*}=\alpha$ with $\alpha<1$, and where the fluctuating line model is assumed to take over. Subsequently, we estimate

$$
\delta^{*}=\left(\frac{\Delta K_{c}}{\alpha K^{\prime}}\right)^{1 /\left(1-\zeta_{\|}^{-}\right)} \delta_{c}^{-\zeta_{\|}^{-} /\left(1-\zeta_{\|}^{-}\right)} .
$$

This length scale $\delta^{*}$ is different to the Larkin length 27] separating various pinning regimes [28] of an elastic line in a random medium. It rather corresponds to the onset of steep front slopes or overhangs (Fig.1) leading to deviations to the Gaussian and mono-affine scaling of the fronts (Fig.3), and therefore limiting the range of validity of the elastic line models. In Fig. 4 we plot $\sigma\left(\delta^{*}\right) / \delta^{*}=\alpha$ for $\alpha=0.35$ and check that it accounts for the crossover between the two scaling regimes for the various experiments performed in different conditions. We conclude that the crossover length scale $\delta^{*}$ is a function of the asperity size $\delta_{c}$, the toughness fluctuations $\Delta K_{c}$ and the stress intensity factor gradient $K^{\prime}$. Following the above arguments, the measure of the crossover $\delta^{*}$ provides an estimate of the link between the magnitude of the toughness fluctuations $\Delta K_{c}$ and the asperity size $\delta_{c}$ knowing the loading conditions $K^{\prime}$. The crossover $\delta^{*}$ depends on $\Delta K_{c}$ and $K^{\prime}$ through a power law with a positive exponent $1 /\left(1-\zeta_{\|}^{-}\right) \approx 5 / 2$, very different from its variation with $\delta_{c},-\zeta_{\|}^{-} /\left(1-\zeta_{\|}^{-}\right) \approx-3 / 2$. Also the crossover $\delta^{*}$ is not expected to scale linearly with the asperity size $\delta_{c}$ as suggested in [29] except if the toughness fluctuations are proportional to the asperity size: $\Delta K_{c} \propto \delta_{c}$. 
Disorder effect - In order to check the effect of disorder and material microstructure (i.e. $\Delta K_{c}$ and $\delta_{c}$ ), we modified the heterogeneities of the sintered interface between the two Plexiglas plates by preparing different samples using glass beads of different diameters $\varnothing_{1} \sim 50 \mu \mathrm{m}$, $\varnothing_{2} \sim 200 \mu \mathrm{m}$ and $\varnothing_{3} \sim 300 \mu \mathrm{m}$. We show in Fig. 4 the

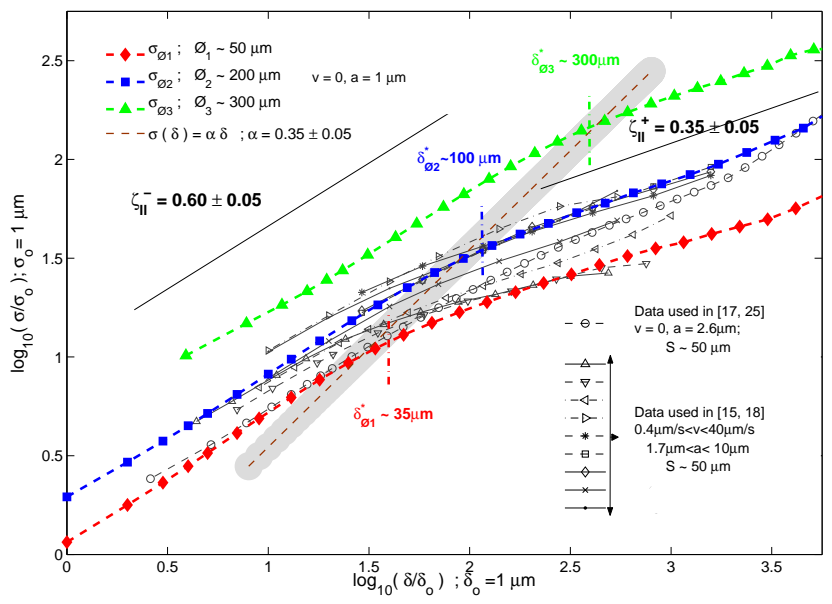

FIG. 4: Effect of disorder on the scaling of interfacial crack fronts. We plot the rms $\sigma$ of the height fluctuations $\Delta y$ as a function of the scale $\delta$ for crack fronts at rest and samples blasted with glass beads of various diameters $\varnothing_{1} \sim 50 \mu \mathrm{m}$, $\varnothing_{2} \sim 200 \mu \mathrm{m}$ and $\varnothing_{3} \sim 300 \mu \mathrm{m}$. The line $\sigma(\delta)=0.35 \delta$ separates the two scaling regimes. To insist on the robustness of our results, we add various data obtained during previous experiments with many different experimental conditions: glassaluminium powder with a typical size around $S \sim 50 \mu \mathrm{m}$ and crack front propagating at various velocities $v[16,18,19,26]$.

scaling behaviour of the interfacial crack fronts with those various types of disorder that influence the toughness fluctuations (unfortunately the measurement of the link between sand-blasting particle size and toughness fluctuations was not possible). We observe mainly the same features as in previous figures with the two different scaling behaviours separated by a characteristic size respectively $\delta_{\varnothing_{1}}^{*}, \delta_{\varnothing_{2}}^{*}$ and $\delta_{\varnothing_{3}}^{*}$. For instance, when using smaller glass beads $\left(\varnothing_{1} \sim 50 \mu \mathrm{m}\right)$, the amplitude of the height fluctuations of the fronts decreases (vertical shift) as well as the scaling range at small scales providing a roughness exponent $\zeta_{\|}^{-} \sim 0.6$ up to the scale $\delta_{\varnothing_{1}}^{*} \sim 35 \mu \mathrm{m}$. We checked that all observations are independent of the sampling resolution, and the analysis techniques (see Fig. 2 and Fig.

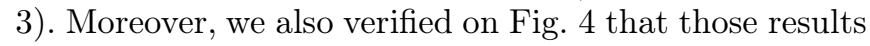
are consistent with the morphology of planar cracks obtained during previous experiments [16, 18, 19, 26] with various conditions concerning both the sample preparation (glass beads mixed with an aluminum powder with a wider size distribution) and the fracturing process with both crack front at rest $(v=0)$ or propagating at various velocities $(0.4 \mu \mathrm{m} / \mathrm{s}<v<40 \mu \mathrm{m} / \mathrm{s})$.

Conclusion - We have analyzed the scaling properties of long planar crack fronts moving along a rough interface between two sintered Plexiglas plates. We identified two scaling regimes separated by a length scale $\delta^{*}$ that depends on the ratio of the local stress drop and the local toughness disorder. Above $\delta^{*}$, the fronts are mono-affine, characterized by a roughness $\zeta_{\|}^{+}=0.35 \pm 0.05$, consistent with the fluctuating line model. Below $\delta^{*}$, we see a different scaling regime, multi-affine, with a roughness exponent $\zeta_{\|}^{-}=0.60 \pm 0.05$. The later roughness exponent is in agreement with the coalescence model. A similar picture may explain observations for the scaling of crack surfaces [7, 29 31] suggesting that crack coalescence is the mechanism operating at small scales corresponding to the process zone while the fluctuations of the elastic front line is the dominating one at larger scales.

We thank D. Bonamy, E. Bouchaud, J. Mathiesen, S. Roux, L. Vanel, M. Adda-Bedia, C. Marlière, S. Zapperi, M. Alava and L. Laurson for helpful discussions.

[1] B. B. Mandelbrot, D. E. Passoja, and A. J. Paullay, Nature, 308, 721 (1984).

[2] E. Bouchaud, J. Phys. Condens. Matter, 9, 4319 (1997).

[3] M. K. Alava, P. K. V. V. Nukala and S. Zapperi, Adv. Phys. 55, 349 (2006).

[4] E. Bouchaud, et al Europhys. Lett. 13, 73 (1990).

[5] K. J. Måløy, et al Phys. Rev. Lett. 68, 213 (1992).

[6] F. Renard, et al, Geophys. Res. Lett., 33:L04 305, 2006.

[7] D. Bonamy et al, Phys. Rev. Lett. 97, 135504 (2006).

[8] L. Ponson et al, Phys. Rev. Lett. 97, 125501 (2006).

[9] A. Hansen, et al Phys. Rev. Lett. 66, 2476 (1991).

[10] V. I. Räisänen, et al Phys. Rev. Lett. 80, 328 (1998).

[11] J. P. Bouchaud, et al, Phys. Rev. Lett. 71, 2240 (1993).

[12] P. Daguier, et al Europhys. Lett. 31, 367 (1995).

[13] J. Schmittbuhl, et al Phys. Rev. Lett. 74, 1787 (1995).

[14] A. Rosso and W. Krauth, Phys. Rev. E 65, R025101 (2002).

[15] D. Bonamy,et al Phys. Rev. Lett. 101, 045501 (2008).

[16] K. J. Måløy et al, Phys. Rev. Lett. 96, 045501 (2006).

[17] J. Schmittbuhl and K. J. Måløy, Phys. Rev. Lett. 78, 3888 (1997).

[18] A. Delaplace et al, Phys. Rev. E 60, 1337 (1999).

[19] K. J. Måløy and J. Schmittbuhl, Phys. Rev. Lett. 87, 105502 (2001).

[20] E. Bouchaud, et al, J. Mech. Phys. Sol. 50, 1703 (2002).

[21] A. Hansen and J. Schmittbuhl, Phys. Rev. Lett. 90, 045504 (2003).

[22] J. Schmittbuhl, et al 90, 045505 (2003).

[23] M. K. Alava and S. Zapperi, Phys. Rev. Lett. 92, 049601 (2004).

[24] J. Schmittbuhl, et al 92, 049602 (2004).

[25] A. Hansen et al, Phys. Rev. E 75, 03102 (2007).

[26] S. Santucci et al, Phys. Rev. E 75, 016104 (2007).

[27] Blatter et al, Rev. Mod. Phys, 66, 1125 (1994).

[28] A. Tanguy and T. Vettorel, Eur. Phys. J. B 38, 71-82 (2004).

[29] D. Dalmas et al, Phys. Rev. Lett. 101, 255501 (2008.

[30] S. Morel et al, Phys. Rev. E 78, 016112 (2008).

[31] P. Daguier et al, Phys. Rev. Lett. 78, 1062 (1997). 
[32] E. Katzav, M. Adda-Bedia and B. Derrida, E.P.L. 78, 46006 (2007). 